\title{
Aromaticity-Controlled Tautomerism and Resonance-Assisted Hydrogen Bonding in Heterocyclic Enaminone-Iminoenol Systems
}

\author{
Roman I. Zubatyuk, Yulian M. Volovenko, Oleg V. Shishkin, Leonid Gorb, \\ and Jerzy Leszczynski
}

Supporting Information

\section{Table of Contents}

Table S1 Total electronic ( $E_{\text {tot }}$ ) and zero-point vibration energies (ZPVE) in Hartree for molecules 1-10 - enaminone (NH) and inimoenol (OH) tautomers.

Table S2 Total electronic ( $\left.E_{\text {tot }}\right)$ and zero-point vibration energies (ZPVE) in Hartree for S3 molecules 1M-10M - aminomethylene (AM) and iminomethyl (IM) tautomers. 
Table S1. Total electronic ( $\left.E_{\text {tot }}\right)$ and zero-point vibration energies (ZPVE) in Hartree for molecules 1-10 - enaminone $(\mathrm{NH})$ and inimoenol $(\mathrm{OH})$ tautomers.

\begin{tabular}{|c|c|c|c|c|}
\hline & \multicolumn{2}{|c|}{ MP2/AUG-cc-pVDZ } & \multirow{2}{*}{$\begin{array}{l}\text { MP4/AUG-cc- } \\
\text { pVDZ//MP2/AUG-cc-pVDZ } \\
\mathrm{E}_{\text {tot }}\end{array}$} & \multirow{2}{*}{$\begin{array}{l}\text { MP2/AUG-cc- } \\
\text { pVTZ//MP2/AUG-cc-pVDZ } \\
E_{\text {tot }}\end{array}$} \\
\hline & $\mathrm{E}_{\text {tot }}$ & ZPVE & & \\
\hline $1 \mathrm{NH}$ & -363.007299 & 0.144431 & -363.138926 & -363.337445 \\
\hline $10 H$ & -363.001817 & 0.144164 & -363.133023 & -363.332511 \\
\hline $2 \mathrm{NH}$ & -435.669957 & 0.100754 & -435.800482 & -436.048599 \\
\hline $2 \mathrm{OH}$ & -435.653387 & 0.101002 & -435.784176 & -436.032559 \\
\hline 3NH & -435.690951 & 0.101238 & -435.821449 & -436.070257 \\
\hline $3 \mathrm{OH}$ & -435.669422 & 0.100937 & -435.800237 & -436.049241 \\
\hline $4 \mathrm{NH}$ & -377.841300 & 0.108330 & -377.956270 & -378.177230 \\
\hline $4 \mathrm{OH}$ & -377.853748 & 0.109617 & -377.967176 & -378.189873 \\
\hline $5 \mathrm{NH}$ & -399.809497 & 0.126164 & -399.942097 & -400.164983 \\
\hline $5 \mathrm{OH}$ & -399.818030 & 0.127131 & -399.949531 & -400.173938 \\
\hline $6 \mathrm{NH}$ & -490.930790 & 0.119686 & -491.073771 & -491.360471 \\
\hline $6 \mathrm{OH}$ & -490.932692 & 0.120323 & -491.075106 & -491.362669 \\
\hline 7NH & -490.924929 & 0.119541 & -491.068209 & -491.354588 \\
\hline $7 \mathrm{OH}$ & -490.920420 & 0.119727 & -491.063082 & -491.350561 \\
\hline $8 \mathrm{NH}$ & -490.918447 & 0.119393 & -491.060896 & -491.347588 \\
\hline $8 \mathrm{OH}$ & -490.921251 & 0.119904 & -491.061435 & -491.350475 \\
\hline $9 \mathrm{NH}$ & -582.076709 & 0.113020 & -582.227082 & -582.581313 \\
\hline $9 \mathrm{OH}$ & -582.072265 & 0.113448 & -582.222747 & -582.577361 \\
\hline $10 \mathrm{NH}$ & -454.993015 & 0.143077 & -455.144904 & -455.398212 \\
\hline $10 \mathrm{OH}$ & -454.987217 & 0.142812 & -455.138169 & -455.392087 \\
\hline
\end{tabular}


Table S2. Total electronic ( $E_{\text {tot }}$ ) and zero-point vibration energies (ZPVE) in Hartree for molecules 1M10M - aminomethylene (AM) and iminomethyl (IM) tautomers.

\begin{tabular}{|c|c|c|c|c|}
\hline & \multicolumn{2}{|c|}{ MP2/AUG-cc-pVDZ } & \multirow{2}{*}{$\begin{array}{l}\text { MP4/AUG-cc- } \\
\text { pVDZ//MP2/AUG-cc-pVDZ } \\
\mathrm{E}_{\text {tot }}\end{array}$} & \multirow{2}{*}{$\begin{array}{l}\text { MP2/AUG-cc- } \\
\text { pVTZ//MP2/AUG-cc-pVDZ } \\
E_{\text {tot }}\end{array}$} \\
\hline & $\mathrm{E}_{\text {tot }}$ & ZPVE & & \\
\hline $1 \mathrm{AM}$ & -249.925639 & 0.133800 & -250.033071 & -250.162063 \\
\hline $1 \mathrm{IM}$ & -249.940679 & 0.133001 & -250.049964 & -250.175329 \\
\hline 2AM & -322.600402 & 0.090487 & -322.705756 & -322.885725 \\
\hline 2IM & -322.590855 & 0.089553 & -322.699761 & -322.873686 \\
\hline 3AM & -322.623419 & 0.091065 & -322.727999 & -322.909027 \\
\hline 3IM & -322.604498 & 0.089767 & -322.713074 & -322.887587 \\
\hline 4AM & -264.742175 & 0.096765 & -264.834885 & -264.984578 \\
\hline $4 \mathrm{IM}$ & -264.794835 & 0.098464 & -264.885812 & -265.034811 \\
\hline $5 \mathrm{AM}$ & -286.715055 & 0.115370 & -286.825426 & -286.977093 \\
\hline $5 \mathrm{IM}$ & -286.758813 & 0.115870 & -286.867668 & -287.018814 \\
\hline $6 \mathrm{AM}$ & -377.844238 & 0.108705 & -377.962983 & -378.180587 \\
\hline $6 \mathrm{IM}$ & -377.873813 & 0.109226 & -377.993509 & -378.207674 \\
\hline 7AM & -377.844238 & 0.108705 & -377.962983 & -378.180587 \\
\hline 7IM & -377.858633 & 0.108673 & -377.978768 & -378.192647 \\
\hline 8AM & -377.834276 & 0.108424 & -377.953626 & -378.170419 \\
\hline 8IM & -377.861360 & 0.108825 & -377.979724 & -378.194694 \\
\hline $9 \mathrm{AM}$ & -468.999807 & 0.102392 & -469.125215 & -469.411146 \\
\hline 9IM & -469.011551 & 0.102370 & -469.139611 & -469.420470 \\
\hline $10 \mathrm{AM}$ & -341.911012 & 0.132227 & -342.037853 & -342.222704 \\
\hline 10IM & -341.926480 & 0.131979 & -342.055104 & -342.235485 \\
\hline
\end{tabular}

